\title{
PARMENIDES E IN PARMENIDEM. LA RECEPCIÓN DEL DIÁLOGO PLATÓNICO Y LA DE SUS INTÉRPRETES TARDOANTIGUOS EN LA DOCTRINA CUSANA DE LA NEGACIÓN
}

\section{PARMENIDES AND IN PARMENIDEM. THE DIALOGUE AND ITS RECEPTION IN LATE ANTIQUITY WITHIN THE CUSAN DOCTRINE OF NEGATION}

\author{
CLAUDIA D'AMICO* \\ Dra. en Filosofía \\ Universidad de Buenos Aires - Buenos Aires - Argentina \\ Círculo de Estudios Cusanos de Buenos Aires
}

Artículo recibido el 30 de julio de 2020; aceptado el o9 de octubre de 2020.

"claudiadamico@yahoo.com.ar

https://orcid.org/oooo-0003-4388-3133

Cómo citar este artículo:

C. D’Amico. (2020). "Parmenides e In Parmenidem. La recepción del diálogo platónico y la de sus intérpretes tardoantiguos en la doctrina cusana de la negación” en Palabra y Razón. Revista de Teología, Filosofía y Ciencias de la Religión. № I8 Diciembre 2020, pp I05-I20 https://doi.org/I0.29035/ pyr.I8.IO5 


\title{
RESUMEN
}

El propósito del artículo es la ubicación de Nicolás de Cusa como parte de la tradición exegética del diálogo Parménides de Platón. Su primer acceso al tema de la negación en el diálogo platónico fue indirecto: a través del corpus areopagiticum y del Comentario de Proclo en la versión de Guillermo de Moerbeke. Luego de la lectura del comentario encarga una versión latina completa del diálogo al célebre aristotélico Jorge Trebisonda. Esa traducción llega a sus manos antes de la redacción del De non aliud y será decisiva para la redacción de esta obra en tanto reformula el vocabulario relativo a la alteridad como modo de la negación. La originalidad de este escrito cusano consiste en la presentación de una fórmula doblemente negativa, inspirada en el Parménides y en sus derivas dionisiana y procleana. Así "non aliud" no es entendido como opuesto a "aliud" sino más bien como la oposición de los opuestos sin oposición.

Palabras claves: Cusano / Platón / Proclo / Dionisio / negación

\begin{abstract}
The purpose of the paper is to locate Nicholas of Cusa as part of the exegetical tradition of Plato's Parmenides dialogue. His first access to the topic of negation in Platonic dialogue was indirect, through the corpus Areopagiticum and Proclus' Commentary in the Latin version done by William of Moerbeke. After reading the Commentary, he asked the famous Aristotelian George of Trebisonda to make a Latin version of the whole dialogue. He read this translation before writing De non aliud and it proved decisive for the writing of that work because it reformulates the vocabulary related to otherness as a mode of negation. The originality of this Cusan text consists in the presentation of a double negative formula, inspired by the dialogue Parmenides and its Dionysian and Proclean interpretations. Thus "non aliud" is not understood as opposed to "aliud" but rather as the opposition of the opposites without opposition.
\end{abstract}

Keywords: Cusanus / Plato / Proclo / Dionysus / denial 
En los estudios sobre los textos de la Antigüedad tardía y el Medioevo se ha discutido en las últimas décadas qué debe entenderse por una "tradición exegética". ${ }^{\mathrm{C}}$ Con más o menos foco en la cuestión metodológica, hay cierto consenso en afirmar que una tradición exegética se construye con un cuerpo de comentarios o bien de menciones y reapropiaciones de una misma obra. Sería enorme la lista de textos clásicos a considerar que inauguraron una tradición, las Categorías o la Metafísica de Aristóteles, la Isagogé de Porfirio, el anónimo Liber de Causis. En esa lista de fundamentales no puede faltar el diálogo Parménides de Platón.

Sabemos que las vías de recepción del platonismo son múltiples y muy variadas en la Edad Media. En el caso particular del Cusano, esos caminos confluyen y han sido estudiados con bastante detalle. ${ }^{2}$ Con todo, el propósito del presente artículo es específico: de un lado, ubicar a Nicolás de Cusa como parte de la tradición exegética de un diálogo particular, Parménides; de otro, mostrar las consecuencias de la reapropiación de su vocabulario recibido a través del comentario procleano pero también por la lectura directa del mismo para la constitución de la terminología relativa a la negación particularmente utilizada en el tetrálogo De ly non aliud de I462.

\section{I) El Cusano y la negación: todos los caminos conducen al Parménides}

La vía de la negación está presente en toda la obra del Cusano con algunos matices y mayor o menor intensidad. Las menciones a Dionisio desde el Sermón I de I430 y la referencia a "verdades negativas" en el primer libro de De docta ignorantia a lo que siguen la cita explícita de tres obras del Areopagita lo ponen de manifiesto. Sumemos a esto la valoración de las teologías afirmativa y negativa en ese mismo libro y la noción de coincidentia oppositorum.

Si buscamos las fuentes del planteo dionisiano acerca del discurso teológico hay una ruta directa al Parménides como ha sido profusamente

\footnotetext{
I Cf. Goulet-Cazé, M.-O. (ed.) (200o) Le commentaire entre tradition et innovation. Actes du Colloque international de l'Institut des traditions textuelles (Paris et Villejuif, 22-25 septembre 1999), Paris, Vrin. Ver también, por ejemplo, A. Bastiaensen (1990) « La perdrix animal méchant figure du diable: Augustin héritier d'une tradition exégétique » dans Augustiniana, t. 40, p. 193-217 y C. D’Ancona (2000), "Syrianus dans la tradition exégétique de la Métaphysique d'Aristote », in Goulet-Cazé, p. $31 \mathrm{II}-327$.

2 Se mencionan solo algunos de los trabajos más recientes que consignan una considerable lista bibliográfica: S. Gersh (ed.) (2014) Interpreting Proclus. From Antiquity to the Renaissance. Cambridge: Cambridge University Press; Secchi, P. (2018), "Cusano e Proclo: la mediazioni platoniche" en Historia Philosophica, I6, pp. 19-45; D'Amico, C. (2019) "Plato and the Platonic Tradition in the Philosophy of Nicholas of Cusa" en Alan Kim (ed.) Brill's Companion to German Platonism, Series: Brill's Companions to Classical Reception, pp. 15-42.
} 
demostrado por el fundacional estudio de Corsini confirmado en investigaciones posteriores. ${ }^{3}$

Las teologías apofática y catafática encuentran su raíz en las primeras hipótesis acerca de lo uno presentadas por Platón. Sin embargo, Dionisio, acaso siguiendo al platónico Proclo que se dedicó especialmente a la primera y allí detuvo su comentario, da un paso más. Su teología apofática radicaliza la vía negativa pues no se trata de la negación que se opone a la afirmación, sino de la negación tanto de afirmaciones como de negaciones. ${ }^{4}$

Podríamos preguntarnos ¿Cuál es la potencia de esa primera hipótesis en el diálogo platónico para que su deriva haya sido tan decisiva? Cómo afirma el clásico libro de Conrford sobre Parménides y Platón, la clave de esta parte del diálogo está en los términos "uno" (hen) y "ser" (to on, einai, ousía) los cuales se muestran "peligrosamente ambiguos". ${ }^{5}$ Para Cornford el propio Aristóteles (participante ficcional del diálogo) aprendió aquí que ser y uno "se dicen de muchas maneras". Pero la genialidad de Platón se encuentra en que la discusión se da sobre esas ambigüedades y, a diferencia de lo que hará Aristóteles, que ofrecerá solo un catálogo de significaciones, Platón se entretiene ejercitando dialécticamente con la ambigüedad. Proclo, que ocupa un papel decisivo en la tradición exegética del diálogo, parece entender lo que pretendía Platón. Cuando comienza el comentario de las hipótesis dice precisamente que si Platón propuso nueve es justamente porque supo que los términos "uno" y "ser" poseen más de un sentido. Sin embargo, comenta solo la primera a la que dedica los dos últimos libros de su comentario.

En esa primera hipótesis Platón expresa que si el uno es uno es no-múltiple y a partir de allí hace que se sucedan las notas negativas. Este Uno no-múltiple será entendido por Proclo como lo Uno exaltado, absoluto o simplísimo. Y mientras Platón dedica 6 o 7 folios a los enunciados negativos de esta primera hipótesis. Proclo dedica casi 200

3 Cf. Corsini, E. (1962) Il Trattato De Divinis nominibus Dello Pseudo-Doinigi e i Commenti Neoplatonici al Parmenide. Univeristà di Torino Pubblicazioni della Facoltà di Lettere e Filosofia XII,4. Torino: Giappichelli. Algunos más recientes, por ej. Andrew Radde-Gallwitz (2010) "Pseudo-Dionysius, the Parmenides, and the Problem of Contradiction" 243-254 en Turner-Corrigan, Plato's Parmenides and its heritage. It's Reception in Neoplatonic, Jewish and Christian Texts, Atlanta, Society of Biblical Literature (vol.2).

4 Williams, J. N. (1999) The Apophatic Theology of Dionysius the Pseudo-Areopagite. Downside Review II7:I57-72.

5 Cornford, F.M. (1989) Platón y Parménides, Madrid, Visor. pp.I74-I75 (primera edición: London, 1939). 
folios a su Comentario: los libros VI y VII se ocupan con exclusividad de la primera hipótesis y en estas páginas se encuentran los pasajes más ricos acerca de la negación.

\section{2) El Cusano frente a los textos}

El Cusano conoció primero el Comentario de Proclo traducido por Guillermo de Moerbeke en el siglo XIII y recién después el texto de Platón por separado. Antes de I440 tiene acceso a unos breves pasajes del libro VI del Comentario que se encontraban en un Codicilo de Estrasburgo que pasó por sus manos. Allí pudo leer la noción de unum exaltatum más allá de toda oposición: no es difícil pensar que apenas lo leyera lo vinculara con Dionisio. ${ }^{6}$

Hay tres menciones al Parménides anteriores a I450, es decir cuando solo conocía los breves pasajes del Codicilo, todas en relación con el tema de la unidad, central en la obra cusana. Desde De docta ignorantia afirma que "la abundancia conviene a lo uno". ${ }^{7}$ Con todo, la reflexión acerca de lo uno o henología se profundiza a partir de De coniecturis. En el Sermón LXXI de I446 el Cusano declara la correspondencia entre la doctrina cristiana de Dionisio y Boecio con la de los "seguidores del Parménides". Los puntos en común que señala en esa ocasión son ciertamente muy generales pero contundentes: todos han sostenido la procedencia de todo a partir de lo uno, lo uno como absoluta necesidad, causa y razón de todo. Todos ellos han comprendido también que lo uno en cuanto causa es complicatio omnium. ${ }^{8}$

La segunda mención puede inferirse en De genesi cuando se refiere a los "platonici". Éste es un caso muy particular pues el Cusano propone un vocablo para denominar a lo absoluto que considera mejor que "unum", tal vocablo es "idem" que destaca precisamente el carácter simplísimo de lo uno mismo. En De Genesi Nicolás sostiene que los platónicos

\footnotetext{
6 Haubst, R. (196I) "Die Thomas- und Proklos-Exzerpte des 'Nicolaus Treverensis' in Codicillus Strassburg 84" in: Mitteilungen und Forschungsbeiträge der Cusanus-Gesselschaft I, pp.I7-5I. He tratado con más profundidad sobre las etapas de la recepción de los textos y las doctrinas procleanas en C. D’Amico (2009) “La recepción del pensamiento de Proclo en la obra de Nicolás de Cusa" en Anales del Seminario de Historia de la Filosofía, XXVI, pp. IO7-I34.

7 De docta ign. (h I n. 5): "Habundantia vero uni convenit".

8 Sermo LXX (h XVI n. 9): "Et nunc poterimus, velut docti ex hac Christi doctrina, omnium philosophorum profundissimos labores, qui videntur studentibus perdifficiles, cum facili explanatione capere. Platonici etenim, prout ex libro Parmenidis Platonis liquet, a multitudine se avertentes ad unum se contulerunt, quod quidem unum est ipsa absoluta necessitas et ratio omnium, quae sunt: Omnia enim in tantum sunt, in quantum unum sunt, ut ait Boethius De unitate et uno. Et absolute unum est «omnis uniter», ut ait Dionysius circa finem De divinis nominibus. Complicat enim omnia ut causa".
} 
se equivocaron en no considerar ningún vocablo mejor a "uno". ${ }^{9} \mathrm{El}$ tratamiento del tema de la identidad en relación con la unidad es un antecedente directo de la temática de De non aliud..$^{10}$ Podría pensarse que hasta aquí el vocabulario cusano es afirmativo, sin embargo se prefigura la noción de una identidad considerada como no-alteridad.

Por último, hay una referencia en la Apologia doctae ignorantiae en alusión a su oponente Iohannes Wenck quien en la sexta conclusión había atacado al Parménides y su noción de unidad absoluta, con esto, atacaba también a todos los doctos y santos teólogos como Dionisio en su Teología mística quien dice que cuando se considera a Dios en su infinitud se lo hace por negación y en este sentido no es ni Padre ni Hijo ni Espíritu Santo."

De este modo, vemos que antes de la recepción del Comentario procleano el núcleo de las menciones al Parménides está constituido por el tema de la unidad, la identidad y la negación. Esto se profundizará primero con la recepción del Comentario completo, luego con la recepción de la nueva traducción del diálogo platónico.

Solo después del 50, el Cusano posee su propio ejemplar completo de In Parmenidem procleano traducido por Moerbeke. Lo mandó a copiar y, como sabemos, lo llena de notas marginales. Las notas sobre las que quiero llamar la atención en esta ocasión son las que remarcan la prioridad de la negación por sobre la afirmación que se encuentran sobre todo en las márgenes de los libros VI y VII que, como dijimos, Proclo dedica al comentario de la primera hipótesis. ${ }^{12}$ Hay una extensa marginal que llama la atención pues el Cusano se tomó el trabajo de

9 De gen. (h. IV n. I45): "Nicolaus: Volo etiam ut attendas quomodo deus alibi vocatur unus et idem. Nam qui virtutibus vocabulorum diligentius operam impertiti sunt, adhuc ipsi idem unum praetulerunt, quasi identitas sit minus uno. Omne enim idem unum est et non e converso. Illi etiam et ens et aeternum et quidquid non-unum post unum simplex considerarunt, ita Platonici maxime".

Io Sobre la vinculación entre "idem" y “non-aliud”, ver W. Beierwaltes (I980) Identität und Differenz, Frankfurt am Main, pp. II4-I2O.

II Apol. (h II n.48): "Ubi vero in sexta conclusione Parmenidem impugnat, non illum tantum, sed omnes doctos et sanctos theologos impugnare nititur, quos nequaquam intelligit; de quo supra [...] Bene habetur, quomodo secundum considerationem infinitatis Deus neque Pater est neque Filius, quia per negationem est consideratio de Deo secundum infinitatem; et ideo omnia tunc negantur, prout etiam sanctus Dionysius in fine Mysticae theologiae per eadem verba hoc idem determinat". Apol. (h II p. Io, nota 9): "Non PLATO Parmen, sed PROCLUS Comment.in Parmen. Hanc rationem ac viam initii...". Cf. R, Klibansky (I929): Ein Proklosfund und seine Bedeutung (Sitzungsberichte der Heidelberger Akademie der Wissenschaften, Philosophisch-historiche Klasse, 5Abhandlung), pp.II-I3.

I2 Cf. Marginales al libro VI: n. 422, n.434, n.494, n.497, n.498, n.5I8, n.520 (CT III, 2.2 p. I06, I09, I23, I24, I28, I29); Marginales al libro VII: n.530, n.535, n.585, n.6I6 (CT III 2.2 p.I3I, I32, I44, I52-3). 
reproducir el texto de Platón que traía el comentario. Se trata de las dobles negaciones, decisivas para Proclo pero también la inspiración directa del último capítulo de la Teología Mística de Dionisio.

"que negantur ab uno: quod non multa, quod neque totum neque partem habens, quod neque principium habet neque medium neque finem, quod nullum habet terminum, quod infiguratum, quod neque in alio est, quod neque movetur, quod neque idem neque alterum, quod neque simile neque dissimile, quod neque equale neque maius neque minus, quod neque senius neque iunius, quod neque generatione neque tempore participat, quod neque esse participat, quod neque nominabile neque decibile, quod neque opinabile neque scibile" (Corresponde a Co II73 ss. ; CT III 2.2, n. 447, pp. II2-3)

La lectura del Comentario y del texto de Platón que él contenía suscitó tanto interés en el Cusano que le pidió al célebre aristotélico Jorge Trebisonda que tradujera completo el diálogo platónico. El llamado "Trapezunzio" lo hizo y dedicó su versión al Cardenal. ${ }^{13}$

Esa traducción llegó a sus manos en 1459 finalizado el De principio, texto en el que trabaja especialmente sobre el comentario de Proclo pero antes de la redacción del De non aliud entre I46I/2. ${ }^{14} \mathrm{Su}$ lectura es decisiva para el tetrálogo. ${ }^{15}$ Esto no fue suficientemente subrayado por mí en la edición de la obra, ahora me permito de algún modo completar el trabajo a partir de la investigación del último tiempo. ${ }^{16}$

Para mostrarlo tomaré dos pasajes centrales de la primera hipótesis y compararemos la versión de Moerbeke contenida en el Comentario y la versión de Trebisonda recibida justo antes de la redacción. El vocabulario es relativo a la identidad y la alteridad.

I3 Cf. Monaco, D. (2012) "Nicolò Cusano e il Parmenide di Platone", in Annuario Filosofico, 28, pp. 479-494. Monaco apunta el siguiente dato: Juan Andrea de Bussi en su prefacio al Didascalico de Apuleyo dice que el Cusano la encargó la traducción, ver esp. pp. 479-480.

I4 Cf. Klibansky, R. et Labowsky, C. (I953) Plato Latinus, vol. III: Parmenides usque ad finem primae hypothesis nec non Procli Commentarium in Parmenidem, pars ultima adhuc inedita interprete Guillelmo de Moerbeka, Londinii. Praefatio. Contiene la traducción de Moerbeke del comentario que adjunta una versión del diálogo extrapolada del texo de Proclo junto con el último libro del comentario de Proclo. La versión de Trebisonda es la primera completa e independiente en latín. Cf. Ruocco,I. (2003) Il Platone latino. Il Parmenide: Giorgio di Trebisonda e il cardinale Cusano, Firenze, Olschki.

I5 Cf. Mónaco (20I2) op.cit., p. 484 n.20) quien tiene razón al atribuir el vocabulario relativo al "non aliud" al desarrrollo de la primera hipótesis (Parm.139b4-139e6) y no de la segunda como afirma Wyller. Cf. Wyller, E. A. (1970) "Zum Begriff "non aliud" bei Nicolaus Cusanus", in Niccolo Cusano agli inizi del mondo moderno, a cura di G. Santinello, Sansoni, Firenze, pp. 419-443.

I6 Cf. Nicolás de Cusa (2008) Acerca de lo no-otro o de la definición que se define a sí misma y a todo, Buenos Aires, Biblos, ver esp. nota 2.4, pp. 359 ss. 
Parmenides $\quad$ I39 b (Moerbeke): Neque etiam idemque alteri neque sibi ipsi erit, neque ru sum alterum neque a se ipso neque $\mathbf{a b}$ altero utique erit.

Parmenides I39 c (Moerbeke): Alterum autem ab altero non erit, donec utique sit unum; non enim uni convenit ab aliquo esse, sed soli alteri $\mathrm{ab}$ altero...
Parmenides $139 \mathrm{~b}$ (Trebisonda): Non erit etiam idem alicui nec aliud ab aliquo nec a seipso et rursus non erit aliud nec a seipso nec ab alio. Parmenides I39 c (Trebisonda): Aliud vero ab alio no erit, dum unum sit. Non enim convenit uni aliud ab aliquo, sed soli alii $\mathbf{a b}$ alio...

Como es evidente, Trebisonda prefiere "aliud" a "alterum". En suma, Platón diría que lo uno bien entendido es no-otro. La consideración semántica de "alter" y "aliud" no arroja diferencias significativas. Pero quizá haya llamado la atención del Cusano que este término utilizado aquí de manera siempre relacional "aliud ab aliquo" o "alii ab alio"... es el mismo de una fórmula que aparece en varias ocasiones en la traducción de Moerbeke y que es frecuentísima en muchos textos: "non aliud quam".

\section{3) De ly non aliud}

Uno de los títulos de la obra De ly non aliud seu directio speculantis encierra la clave de su desarrollo: el "ly" está indicando que "non-aliud" es tomado en cuanto vocablo. Se trata de una de las muchas propuestas cusanas para denominar lo absoluto con un nombre enigmático. Como "aenigma", "non-aliud" es un vocablo que nombra lo que por sí mismo es innombrable y vuelve visible, lo invisible. Este nombre enigmático por su fuerza significativa es una guía (manuductio) para el que especula: una auténtica "directio speculantis". ${ }^{17}$ Para poner de manifiesto tal fuerza significativa, el Cusano recurre al tema de la definición considerada como camino hacia el conocimiento. La argumentación se orienta a mostrar que "non aliud" es el elemento que permanece en toda definición, en tanto que cada cosa es no-otro que sí misma. En este sentido, "non aliud" significa negativamente lo "idem".

Ahora bien, si se busca la definición de la definición, es decir la definición de lo no-otro mismo, resulta de ello la definición que se define

I7 Cf. Dupré, W. (1962) Die Non-aliud Lehre des Nikolaus von Kues, Wien, Diss.; Von Bredow, G. (I965) "Gott der Nichtandere", en Philosophisches Jahrbuch, 73, I, pp. 15-22; Gutwenger, E. (1970) Das "Nichtandere“ bei Nikolaus von Kues. en Cusanus Gedächtnisschrift (Hg. N. Grass) Innsbruck / Wien, pp. 17-22. 
a sí misma y a la vez define todo. ${ }^{18}$ Esta absoluta definittio es llamada también conceptus absolutus. ${ }^{19}$ Se arribará pues a la siguiente fórmula: "no-otro es no-otro que no-otro" (non aliud non aliud quam non aliud).

Así, la definición absoluta revela un carácter trino, en tanto expresa la absoluta relación entre la unidad (non aliud), su igualdad o Verbo (non aliud) y el nexo indisoluble entre ambos (non aliud quam).

Podría pensarse que al llamar "non aliud" no solo a toda la naturaleza divina sino a cada una de las personas trinitarias, el Cusano podría estar desafiando el dogma que precisamente utiliza "alius" para distinguir a las personas de la Trinidad. Pero hay que notar que no se niega el "alius" como adjetivo masculino que califica al Padre, al Hijo y al Espíritu, sino el neutro "aliud" que es la condición de otredad. ${ }^{20}$ Por otra parte, es necesario subrayar que "non-aliud" es tomado en cuanto término y esto se hace evidente no solo por el mencionado uso del "ly" sino también porque no aparece declinado en todo el texto, cualquiera sea su función sintáctiva permanece idéntico.

La definición absoluta es, pues, el principio creador unitrino que al definirse a sí mismo se vuelve medida de todas las cosas (mensura omnium). Medida es, en este caso, el límite y la identidad de cada una de ellas: "el cielo es no-otro que cielo". Sin embargo, esta noción que menciona lo no-otredad presenta una ventaja respecto de "idem". No sólo resulta adecuada para indicar la identidad sino también el principio de diferenciación: "el cielo es otro del no-cielo". Así, "non aliud" es el principio indistinto que realiza la distinción en todos los seres. ${ }^{2 \mathrm{I}}$ Lo no-otro en tanto trascendente a todo, excluye de sí toda alteridad y es

I8 Uno de los manuscritos de la obra lleva por título, Deffinitio omnis deffinientis. Se trata del manuscrito de la Biblioteca Capitular de Toledo. Cf. K. Reinhardt (I986) "Eine bisher unbekannte Handschrift mit Werken des Nikolaus von Kues in der Kapitelsbibliothek von Toledo", en Mitteilungen und Forschungsbeiträge der Cusanus-Gesselschaft I7, pp. 96-I33.

I9 Acerca de la vinculación entre "conceptus absolutus" y "non aliud", Cf. Schwaetzer, H. (20II) "Non aliud" en Nikolaus von Kues, De non aliud. Nichts anderes, Münster, Aschendorff, pp. 203-209.

20 Monaco, D. (2012) p. 49I, n. 5I: Già nel suo primo sermone tramandatoci risalente al I430, In principio erat Verbum, il cardinale tedesco utilizza la negazione dell'aliud in contrapposizione all'affermazione dell'alius per indicare la comunione essenziale delle tre persone divine pur affermandone la distinzione personale (...) Tra le possibili fonti cusaniane la distinzione tra alius e aliud in ambito trinitario è già impiegatada Agostino al fine di affermare la distinzione delle persone e l'unità della sostanza. L'uso della negazione dell'aliud in contrapposizione all'affermazione dell'alius ricorre altresì anche in un'altra fonte fondamentale del cardinale, Meister Eckhart. Il maestro domenicano in più luoghi distingue l'altro secondo la persona, l'alius, dall'altro secondo la natura l'aliud".

2I W. Beierwaltes ha encontrado en esto una inspiración eckhartiana señalando el Sermón IV I (LW IV 28, 5) y la Expos. libri Sap., n. I54 (LW II 490, 8) en los cuales Eckhart llama a Dios indistincta distinctio. Cf. W. Beierwaltes (I980) op.cit., pp. II4-II7. 
anterioridad respecto de todo lo que es otro; pero, al mismo tiempo, está presente como no-otro en todo lo que es otro. ${ }^{22}$

Después de un extenso capítulo en el promedio de la obra en el cual se presenta un florilegio de textos de Dionisio llamado el "máximo de los teólogos"23 las referencias a la tradición platónica se suceden hasta el final.

La larga serie de citas se abre y se cierra con el núcleo del pensamiento de Dionisio en el cual son evidentes las huellas del Parménides: la incomprensibilidad de lo absoluto, presente en todo y por sobre todo, incomprensible e inefable, el cual no es parte ni todo ya que lo comprende todo y está antes de todo. Es la medida de las cosas y es el siglo, por sobre el siglo y antes del siglo. ${ }^{24}$ Así pues, Dios es conocido en todo y separado de todo, tanto por la ciencia como por la ignorancia. Nada hay contrario a Él mismo. ${ }^{25}$ En todo es todo, y en nada, nada. ${ }^{26} \mathrm{~A}$ partir de esta sentencia fundamental, que ha sido suficientemente estudiada por K. Kremer, ${ }^{27}$ se hace evidente el núcleo de lo que el Cusano quiere destacar de la obra del Areopagita: hay algo por sobre lo uno mismo que determina lo que es uno. Tal uno recoge en él todo uniformemente "antes". ${ }^{28}$

\section{4) Unum ante unum. Las fuentes y los interlocutores del diálogo}

Es notable que Nicolás de Cusa destaque en la obra de Dionisio un aspecto que las múltiples lecturas medievales no habían destacado suficientemente: la distinción entre lo uno como opuesto a lo múltiple y lo uno absoluto entendido como anterioridad absoluta. Podríamos, en ese sentido, señalar un único antecedente, Bertoldo de Moosburg, a quien el Cusano menciona en su Apologia como comentador de Proclo

22 Cf. Bormann, K (1973) "Zur Lehre des Nicholaus von Kues von des Andersheit und deren Quellen", in Mitteilungen and Forschungsbeitrage der Cusanus-Gesellschaft, I0, pp. 130-37; Mc Tighe, Th. P. (1990), "Contingentia and Alteritas in Cusa's Metaphysics", in American Catholic Philosophical Quarterly, vol. LXIV, I, pp. 55-7I.

23 Cf. Stallmach, J. (I960) "Das 'Nichtandere' als Begriff des Absoluten. Zur Auswertung der mystischen Theologie des Pseudo-Dionysius durch Cusanus", in Sonderdruck aus Festschrift für Bischof Dr. Albert Stohr, Mainz, pp. 329-335.

24 Cf. De non aliud (h. XIII n. 6o)

25 Cf. De non aliud (h. XIII n. 64)

26 Cf. De non aliud (h. XIII n. 65

${ }_{2}$ Cf. Kremer, K. (1986) "Gott -in Alem Alles, in Nichts Nichts. Bedeutung und Herkunft dieser Lehre des Nikolaus von Kues" en Mitteilungen and Forschungsbeitrage der Cusanus-Gesellschaft, I7, pp. I88-219.

28 Cf. De non aliud (h. XIII n. 70) 
y cuya obra parece conocer. El fin del florilegio conduce a Nicolás de Cusa a la postulación de lo que denomina "Principio A" (Principium A), indicando la misma anterioridad significada por lo "no-otro": lo nootro es antes del mismo "antes". La postulación del Principium A cierra la referencia a Dionisio y, de alguna manera, introduce la referencia a Proclo.

El Cusano afirma que Proclo confirma los dichos de Dionisio "casi con las mismas expresiones", haciéndolos parte de la misma tradición exegética que parte del Parménides. ${ }^{29}$

Si atendemos a los interlocutores del tetrálogo, también hallamos las huellas del diálogo platónico en más de un sentido: el abad se presenta como especialista en ese diálogo y en el Comentario de Proclo, el hecho de que mencione a ambos por separado, implica la consideración de la nueva traducción de Trebisonda; Pedro Balbo se presenta como el reciente traductor de otro texto de Proclo, la Teología Platónica, escrito que se refiere fundamentalmente al Parménides considerándolo la cumbre de tal teología; el Cardenal, que es el propio Cusano, se presenta como especialista en Dionisio, deudor de la misma tradición y Fernando, el joven aristotélico que introduce el tema de la definición, recuerda al joven Aristóteles, personaje del diálogo platónico.

La referencia al diálogo platónico a través de las dos obras de Proclo mencionadas ocupa los últimos cuatro capítulos. Si se atiende a las notas que el Cusano también puso a la traducción de la Teología Platonica de Pedro Balbo se advierte claramente que la lectura directa del Parménides que realizó el Cusano lo condujo a confirmar la lectura procleana.

Para Proclo, el Parménides es la consumación de la "teología platónica" en tanto trata de "los principios primeros que se bastan por completo a sí mismos". ${ }^{30}$ Tales principios son para su propio sistema, lo uno y las hénadas, denominadas "dioses", lo cual justifica por sí mismo el carácter "teológico" de la cuestión tratada. El juego dialéctico platónico está para Proclo al servicio del tema teológico.

Esta exégesis del camino elegido por Platón al comienzo de

\footnotetext{
29 Cf. De non aliud (h. XIII n. 90)

30 Proclus, Theol.plat. II, 3: "unum non aliud quam unum", repetida en marg cus. n. I43 (Cf. Cusanus Texte III (1986) Marginalien.2. Die Exzerpte und Randnoten des Nikolaus von Kues zu den lateinischen Übersetzungen der Proclus, Heidelberg.
} 
Parménides resulta muy afín con la inspiración general de De non aliud. ${ }^{31}$ En efecto, como Platón -al menos en la exégesis procleana-- Nicolás de Cusa para esclarecer la relación uno-múltiple, acude a un ejercicio lógico a partir de las definiciones y a partir de este ejercicio lógico realiza un desarrollo que considera, en sí mismo, teológico.

\section{5) Lo otro y lo no-otro: Non aliud como aliud aliorum- Las Proposiciones}

Provocativamente, en el capítulo 23 el Cusano quiere poner en contradicción a Dionisio con Platón y Proclo recordando que en De divinis nominibus el "máximo teólogo" llamó a Dios "otro". ${ }^{32}$ Sin embargo, rápidamente retoma el camino de la concordancia proponiendo la siguiente fórmula: para todos ellos lo absoluto es "lo otro de todo".

El Cusano pudo leer en las versiones latinas de las obras de Dionisio y Proclo la expresión "alterum omnium" (que traduce el "héteron pánton") y advertir la carga negativa-excesiva de este "Otro". Lo "Otro" es lo "no-múltiple"33, más aún la negación misma de la multiplicidad. Así, este "Otro" que, en el texto cusano, se entiende como lo "no-otro" resulta ser "lo otro de los otros" (aliud aliorum).

Si se piensa bien, "non aliud" y "aliud aliorum" son fórmulas equivalentes. Sin embargo, no son las caras negativa y positiva de lo mismo. Ambas fórmulas son negativas. Ambas ofrecen una negatio negationis como superabundancia de la afirmación y en este sentido su fuerza significativa es superior a la del nombre "unum".

La pretensión de una síntesis fue anticipada de alguna manera por el Cusano en las veinte proposiciones post-puestas a la obra y que fueron consideradas por bastante tiempo como un escrito autónomo. En el año I500, Conradus Celtis, un humanista amigo de Hartmann Schedel, las publicó en Viena bajo el título que aparece en el manuscrito de München: "Propositiones de virtute ipsius non aliud". Las Propositiones contaron con una nueva edición también en Viena en I556. Sin embargo, no tuvieron

3I Cf. Willer, E. (1974) “Nicolaus Cusanus De non aliud und Platons Dialog Parmenides", en Studia Platonica, Amsterdam, pp. 239-25I.

32 Cf. De non aliud (h. XIII n. IOo).

33 Nicolás de Cusa ya había leído la noción procleana de "no-múltiple" en In Parm., VI (Stell p. 375, Co I087): "Si autem est, palam quod non multa est. Non enim utique esset ut vere unum, neque uno repletum; nam multa non unum". También había anotado como marginal (Steel ad p. 375, O0-I): "vere unum non est multa". Sin embargo, la idea de lo uno como "no múltiple" reaparece destacada en la Theol. Plat. 
mucha difusión en la Modernidad. ${ }^{34}$ El hecho de que se lo considerara un texto independiente se basa en que el conjunto de las proposiciones es comprensible por sí mismo sin el auxilio del escrito De non aliud. En ellas se encuentran explicitados los tópicos fundamentales tratados en el escrito, pero a diferencia de este no hay referencia alguna a las fuentes. Es necesario destacar que su modo de enunciación es prescriptivo a la manera de una manuductio. Así, las proposiciones son una auténtica directio speculantis. Esto se confirma si pensamos que todas, sin excepción, aluden a una mente activa. La mayor parte de las proposiciones se inician con fórmulas como "quisquis videt...", "qui videt...", "quidquid mens videt...". De esta manera, se pone en evidencia que todo lo que se afirme de "non aliud" en tanto principio del ser, se corresponde con el hecho de que es también principio del conocer. Todo lo que la mente ve, no lo vería sin lo no-otro. ${ }^{35}$

El título "de virtute ipsius non aliud" destaca por una parte la virtus o fuerza propia de lo absoluto; pero por otra muestra la vis significandi de "non aliud" en cuanto vocablo, expresado nuevamente por la partícula "ly". Reaparece en primer lugar el tema de la definición que se define a sí misma y a todo, como aquello buscado por la mente. ${ }^{36}$ La posibilidad de repetición de lo mismo que ofrece esta fórmula le confiere precisamente su fueza significativa y la convierte en aenigma en tanto permite a la mente "ver". En este sentido, en muchas de las proposiciones se muestra que otros vocablos sólo son definibles por lo no-otro, sin definirse a sí mismos. Así como se mostró la superación por lo "no otro" de la denominación platónica "uno", se muestra también la insuficiencia de otros vocablos que necesitan de lo "no otro" para ser definidos: "principium", "medium", "finis", "nomen", "ens", "non-ens" y así de todos los singulares que pueden ser dichos o pensados. Se reitera que, por esto mismo, lo no-otro es "todo en todo", "singular en lo singular" y "nada en nada". ${ }^{37}$ El Cusano niega también que sea pensamiento, es más bien "pensamiento del pensamiento" (cogitatio cogitationum). ${ }^{38}$ Esta fórmula de raíz aristotélica es aquí equivalente a "definición de la definición", la cual definiéndose a sí misma define todo y es medida adecuada de todo. Esto es la creación: la mostración de la mente divina autodefiniéndose y poniendo límite

34 Cf. S. Meier-Oester, Die Präsenz des Vergessenen. Zur Rezeption der Philosophie des Nicolaus Cusanus vom I5. bis zum I8. Jahrhundert, Münster, 1989, p. 72.

35 Cf. De non aliud (h. XIII n. II6-II7)

36 Cf. De non aliud (h. XIII n. II4)

37 Cf. De non aliud (h. XIII n. II5)

38 Cf. De non aliud (h. XIII n. II6) 
a todo. ${ }^{39}$ Para clarificar presenta imágenes a las que había recurrido en obras anteriores: la creación como una elocución viva para los oyentes más cercanos --las creaturas jerárquicamente más altas-y como un discurso escrito para los más lejanos. Es notable que el Cusano duplique las fórmulas para subrayar que el principio no es solo inmanente sino que trasciende lo principiado. Afirma que es la eternidad de la eterna eternidad, la unidad de la unidad de lo uno, la bondad de la bondad de lo bueno, la entidad de la entidad del ente, etc. que reluce en todo unitrinitariamente..$^{40}$ Esta duplicación resignifica también el sentido de la participación. El principio no es la unidad que se participa en todo uno, sino que es la unidad imparticipable anterior a toda unidad participada.

Para la mente solo es posible ver la identidad participada del aliud por medio de non-aliud. Ahora bien, esa profunda identidad se presenta, a la vez, como coincidencia. Así, lo no-otro permite ver en cada uno de los opuestos al otro opuesto: en la afirmación, la negación afirmada; así como en lo calefaccionado, lo no-calefaccionado calefaccionado. ${ }^{4}$ De este modo, se hacen visibles los aspectos que constituyen todo singular: la determinación y la quididad. Mientras las afirmaciones dirigen la vista hacia el "tale quid", las negaciones dirigen la vista hacia el "quid". ${ }^{42}$ Precisamente por señalar solo el tale quid toda afirmación es insuficiente. ${ }^{43}$ Sin embargo, para el Cusano --como para Proclo y Dionisio-la afirmación también cumple un rol ascensional: dirige la mirada más allá de sí misma, hacia la quididad que es lo no-otro en cada otro. ${ }^{44}$

En suma, De non aliud retoma un tópico que se encuentra en toda la filosofía cusana: lo uno, la identidad y la alteridad. La originalidad de este escrito consiste en la presentación de una fórmula doblemente negativa, inspirada en el Parménides y en sus derivas dionisiana y procleana, en la cual "non aliud" no debe ser entendido como opuesto a "aliud" sino como oposición de los opuestos sin oposición. Así lo dice el Cusano en el

39 Cf. De non aliud (h. XIII n. II8)

40 Cf. De non aliud (h. XIII n. II9)

4I Cf De non aliud (h. XIII n. 53-75) se corresponde con los caps. XIV-XV.

42 Cf. De non aliud (h. XIII n. I2O)

43 Cf. De non aliud (h. XIII n. I2I9

44 Cf. De non aliud (h. XIII n. I25) Se reitera que él es principio del ser y del conocer: Dios da al intelecto el ser no-otro que intelecto que entiende y para lo inteligible el ser no-otro que inteligible y así para las distintas potencias inferiores al intelecto. Sorprendentemente el Cusano cierra la serie de las Propositiones recurriendo al vocabulario aristotélico. Se refiere a la potencia que encuentra su plenitud en el acto y la necesidad de un motor que mueva desde la potencia al acto. Este movimiento natural es necesario pues ninguna potencia puede ser vana. Recurriendo nuevamente a la duplicación, y sin temor a una regressio ad infinitum afirma el Cusano que lo no-otro es natura naturae en la cual toda naturaleza encuentra la plenitud y reposo que le son propios. 
comienzo de la Proposición XVIII con la que cerramos esta presentación:

"Quien ve cómo lo no-otro, que es lo otro de lo otro mismo, no es lo otro mismo, aquel ve lo otro de lo otro mismo, lo cual es lo otro de los otros..." 45

45 De non aliud (h. XIII n. I23): "Decima octava: Qui videt, quomodo >non aliud`, quod est aliud ipsius aliud, non est ipsum aliud, ille videt aliud ipsius aliud, quod est aliud aliorum ..." 
